\title{
Vasarely painting at the nanoscale on sapphire crystals
}

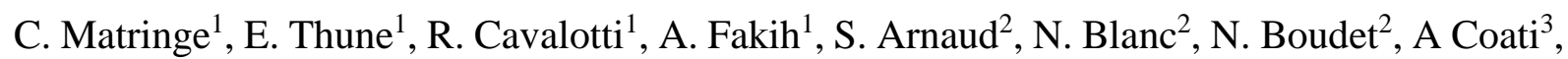
Y. Garreau ${ }^{3,4}$, D. Babonneau ${ }^{5}$, R. Guinebretière ${ }^{1 *}$

${ }^{1}$ Université de Limoges, IRCER, UMR CNRS 7315, Centre Européen de la Céramique, 12 rue Atlantis, 87068 Limoges, France

${ }^{2}$ Université Grenoble Alpes, Institut Néel UPR CNRS 2940, CRG D2AM Beamline, ESRF, 38042 Grenoble, France

${ }^{3}$ Synchrotron SOLEIL, SixS Beamline, BP48, 91192 Gif sur Yvette, France

${ }^{4}$ Université de Paris, Laboratoire Matériaux et Phénomènes Quantiques, UMR CNRS 7162, 75013 Paris, France

${ }^{5}$ Université de Poitiers, Institut Pprime, Département Physique et Mécanique des Matériaux, UPR CNRS 3346, SP2MI, TSA 41123, 86073 Poitiers Cedex 9, France

*Author to whom correspondence should be addressed: rene.guinebretiere@ unilim.fr

\section{TOC}

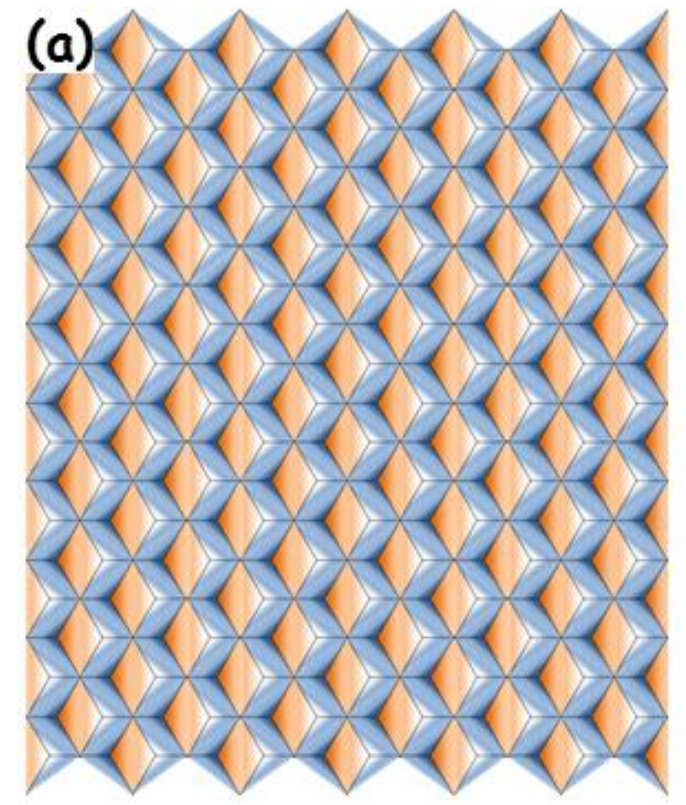

$10^{-7} \mathrm{~m}$

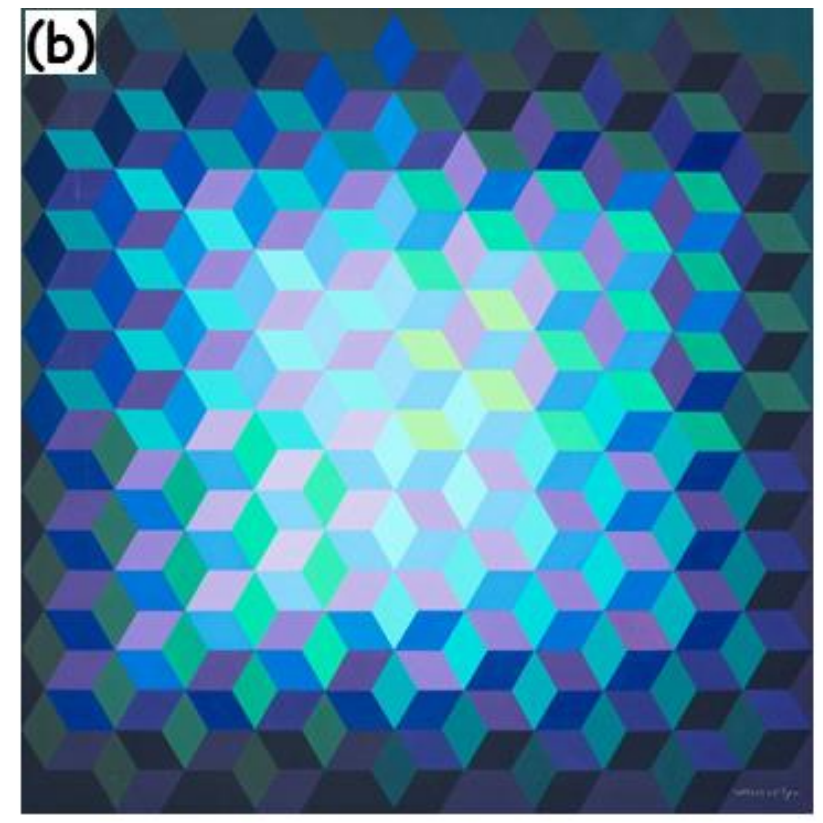

$10^{-1} \mathrm{~m}$ 


\begin{abstract}
We demonstrate that convenient thermal treatment of a specific sapphire vicinal surface can induce the formation of a fully two-dimensional (2D) ordered surface made of a periodic assembly of (006) facets. The similarity between the resulting surface topography and patterns represented in the "Hexagon series" of paintings by Vasarely is really striking! We thus propose to call these surfaces as "nanoscaled Vasarely surfaces". We also show that the selforganisation process, which is driven by the minimisation of the free energy of a closed system, results in a quasi-linear isothermal growth of the facets' surface area over time.
\end{abstract}

Keywords: Nanoscaled Vasarely surfaces, 2D-Self-organization, Sapphire vicinal surfaces

\title{
1. Introduction
}

Scaling down of the electronic or optoelectronic devices often requires the elaboration of twodimensional (2D) periodic structures at the nanoscale. Beside the conventional top-down fabrication technique that consists in etching macroscopic crystals by electron beam writing or advanced lithography using high energy photon beams, self-ordering offers an elegant and low cost bottom-up route [1]. In this field, the use of ordered vicinal surfaces of single crystals is a very efficient way to elaborate periodic templates $[2,3]$. In the case of stable phases, free of any solid-state phase transition or chemical decomposition in the concerned temperature-pressure range, the decrease of the surface free energy drives the out-of-equilibrium continuous selfordering process [3] that is often described as a spinodal decomposition process [4]. Various vicinal surfaces of metals [5], semiconductors [6] or insulating oxides [7,8] constituted by periodically spaced steps have been obtained after convenient thermal treatments, thereby resulting in the formation of one-dimensional (1D) ordered templates at the nanoscale.

Sapphire, single crystal of $\alpha$-alumina, is playing an ever-increasingly important role as a base material for nanoelectronic systems. For example, it is commonly used as substrate for growing 
indium gallium nitride (InGaN) $[9,10]$ as well as zinc oxide ( $\mathrm{ZnO})$ [11,12] epitaxial nanostructures for electroluminescent devices. More generally, sapphire surfaces are used as a substrate for the growth of inorganic [9-16] as well as organic nanostructures [17]. Recent studies have focused on the continuous evolution of the period of the steps formed by isothermal treatment of vicinal sapphire surfaces exhibiting a miscut angle $\alpha_{\mathrm{m}}$ between $1^{\circ}$ and $10^{\circ}$ with respect to the (006) crystallographic planes [18]. The corresponding surfaces are constituted by a 1D periodic arrangement of prisms with a triangular saw tooth-like profile and, in the case of $\alpha_{\mathrm{m}}=10^{\circ}$, a symmetric profile can be obtained [19]. In this article, we show that long isothermal treatment of such a surface induces an astonishing 2D-ordering. The surface can then be described as a 2D periodic assembly of diamond-shaped facets separated from each other by parallelogram-shaped steps. As shown in fig. 1, the resulting surface pattern is very similar to the "Hexagon VII" painting realized by Vasarely and, as far as we know, such a 2D periodic topography at the nanoscale has never been elaborated through self-ordering process. This article is focused on the quantitative description, with respect to crystallographic aspects, of this "nanoscaled Vasarely surface".

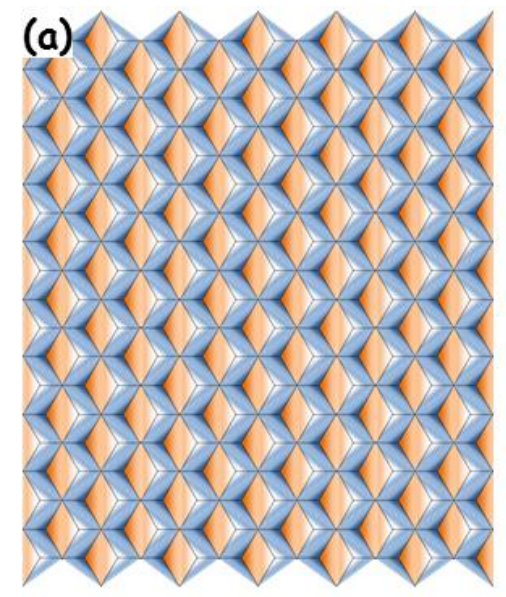

$10^{-7} \mathrm{~m}$

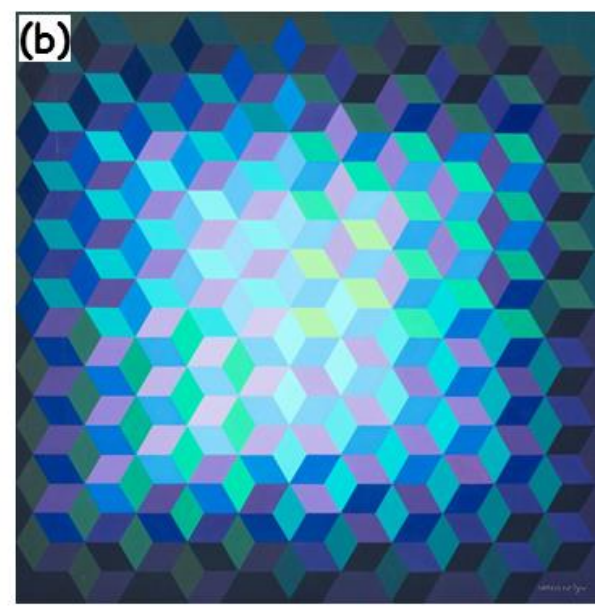

$10^{-1} \mathrm{~m}$

Figure 1 Nanoscaled Vasarely surface on a sapphire crystal. (a) Modelling of the surface from analysis of X-ray scattering measurements. (b) Reproduction of the "Hexagon VII" Vasarely painting realized in 1969.

\section{Experimental}

Sapphire samples, cut with a miscut angle $\alpha_{m}=10^{\circ}$ with respect to the (006) planes and in such a way that the step edges were parallel to the [110] direction, were purchased from the 
MTI corporation Richmond, CA, USA. The 2D-ordering was promoted by annealing through isothermal treatment at $1250{ }^{\circ} \mathrm{C}$ under a $\mathrm{P}_{\mathrm{O} 2}=1$ bar atmosphere.

The morphology of sapphire vicinal surfaces was observed using Atomic Force Microscopy (AFM) with a MultiMode SPM Microscope in tapping mode under ambient atmosphere at room temperature. AFM images were taken with a silicon cantilever with an integrated pyramidal tip of typical radius of curvature smaller than $10 \mathrm{~nm}$ (manufacturer's data). The force constant was $80 \mathrm{~N} / \mathrm{m}$, and the resonant frequency range was from $200 \mathrm{kHz}$ to $400 \mathrm{kHz}$. The topographic images were drawn with the WSxM software [20].

Grazing Incidence X-ray Diffraction (GIXD) experiments were done on the MED instrument of the SixS beamline at the Synchrotron SOLEIL, in Gif-sur-Yvette, France. The samples were irradiated by a $12 \mathrm{keV}$ X-ray beam under a grazing incidence angle equal to $0.30^{\circ}$. The diffracted intensity around several reciprocal space nodes was recorded under in-plane geometry using a point detector.

Grazing Incidence Small-Angle X-ray Scattering (GISAXS) experiments were performed on the BM02 French CRG beamline [21] at the European Synchrotron Radiation Facility (ESRF) in Grenoble, France. All the GISAXS maps were recorded using a monochromatic X-ray beam at $9.659 \mathrm{keV}$ and under a grazing incidence slightly higher than $0.23^{\circ}$ that is the critical angle for total reflection of sapphire at this energy. The GISAXS maps were recorded from samples mounted onto a diffractometer allowing careful sample orientation with respect to the crystallographic axis of the crystal. We used a XPAD solid state detector located $5235 \mathrm{~mm}$ away from the sample and we recorded $\left(\mathrm{q}_{\mathrm{y}}, \mathrm{q}_{\mathrm{z}}\right)$ maps as a function of the azimuthal angle $\varphi$ with a step of $0.5^{\circ}$. Measurement of 721 maps in the full $360^{\circ}$ range allows complete reconstruction of the 3D GISAXS maps. 2D maps were analyzed according to the distorted-wave Born approximation using the FitGISAXS package [22]. According to the classical convention, the $\overrightarrow{\mathrm{q}_{\mathrm{x}}}$ component of the scattering vector $\overrightarrow{\mathrm{q}}$ corresponds to the projection of the X-ray primary 
beam onto the sample surface, the $\overrightarrow{\mathrm{q}_{\mathrm{z}}}$ component is orthogonal to the scattering surface and the $\overrightarrow{\mathrm{q}_{\mathrm{y}}}$ component is orthogonal to the two others.

\section{Results and discussion}

$\alpha$-alumina crystallizes under the $\mathrm{R} \overline{3} \mathrm{c}$ space group. The crystallographic structure of this rhombohedral phase is usually described using a hexagonal multiple cell and, all along the article, this hexagonal indexing will be used. Sapphire exhibits a 3-fold symmetry around the c-axis in the hexagonal setting and the [110], [100] and [010] directions lying in the (006) planes are thus equivalent (see fig. 2a). Our samples were all cut with a miscut angle $\alpha_{\mathrm{m}}$ equal to $10^{\circ}$ with respect to the (006) planes and in such a way that the step edges were parallel to the [110] direction. It is interesting to note that such a cutting is breaking the 3 -fold symmetry around the c-axis and only the mirror perpendicular to the [110] direction is maintained. Selfordering of this sapphire vicinal surface is illustrated in figs. 2b-e by AFM images. After 8 hours of isothermal treatment at $1250{ }^{\circ} \mathrm{C}$ under oxygen, a 1D-ordered surface is obtained (see fig. 2b) as previously fully discussed [19]. The surface presents a periodic faceting with (006) and (119) facets. More unexpectedly, longer thermal treatments promote the nucleation of areas exhibiting a 2D-ordering which are growing with the treatment duration (figs. 2c-d). Finally, after 32 hours, the surface is fully ordered in two directions (fig. 2e), as clearly evidenced by the Fourier transform of the AFM image (see below the AFM image fig. 2e).

The topography of the sapphire vicinal surface heat-treated for 32 hours can be described as an assembly of three-dimensional (3D) objects characterized by a certain shape and periodically spaced from one another. Following the crystallographic general approach, we can describe this ordered surface by a 2D lattice and plane group, with each node of this plane group being decorated by the same 3D motif. Both the plane group and the motif were determined through X-ray scattering experiments, which are able to provide statistical information averaged over macroscopic dimensions. In order to achieve high surface sensitivity, all the experiments were realized under grazing incidence. In the reciprocal space, each node (RSN) associated to the X- 
ray diffraction of such a surface exhibits an intensity distribution that corresponds to the 3DFourier transform of the motif shape. Thus, grazing incidence X-ray diffraction experiments were conducted with the aim of getting full 3D maps of several RSNs. As a typical example, fig. $2 \mathrm{f}$ and $2 \mathrm{~g}$ show $h k$ reciprocal space maps (RSMs) recorded for the $113 \mathrm{RSN}$ at different $l$ values, symmetrically below and above the center of this node. A clear splitting into three spots is observed except for the $h k$ map recorded at $l=3$ (not reported in the figure). At each $l$ value, the 3 observed spots correspond to the intersection of a reciprocal space slice parallel to the $(h, k)$ plane and the crystal truncation rod (CTR) [23] associated to each diffracting facet. First, it clearly appears that whatever the value of $l$, one spot is always located at the $(1,1)$ coordinates in the $(h, k)$ plane. It means that this CTR is parallel to the $\mathrm{c}^{*}$-axis and thus, one of the facets is parallel to the (006) planes. As expected, the (006) facets lying at $10^{\circ}$ from the mean surface are thus conserved throughout the self-ordering process. Considering the position of the two other spots in each $h k$ map, we identified two others CTRs each cutting the 113 RSN at its center. The directions of these CTRs are given by the reciprocal space vectors $(2, \overline{1}, 15)$ and $(1, \overline{2}, 15)$, respectively. The corresponding facets are equivalent with respect to the mirror symmetry of the surface. It is important to underline that similar diffraction patterns (i.e. 3 CTRs crossing at the RSN position) were observed for all the measured RSNs. Finally, these findings demonstrate that the diffracting surface is made of an assembly of 3 types of facets respectively parallel to the (006), $(2 \overline{1} 15)$, and $(1 \overline{2} 15)$ planes. As shown in fig. $2 \mathrm{~h}$, we were able to determine the crystallographic directions corresponding to the intersections of these facets.

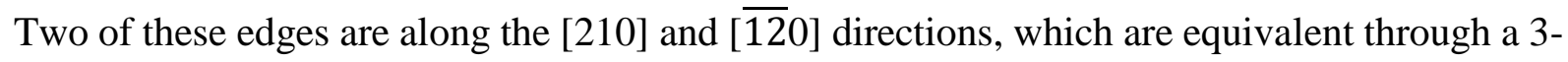
fold rotation with respect to the c-axis in the hexagonal setting and the third edge is parallel to 
the [55 1$]$ direction, which is orthogonal to the in-plane [110] initial sapphire cutting direction and forms an angle of $\gamma_{\mathrm{m}}=17.5^{\circ}$ with respect to the [1 $\left.1 \overline{1} 0\right]$ direction.

This new morphology of the surface is obtained after long thermal treatments of a primary 1Dordered hill-and-valley surface exhibiting (006) and (1 $\overline{1}$ 9) facets; the latter are constituted by a periodic succession of (006) flat terraces separated by steps along the [110] direction (see fig.

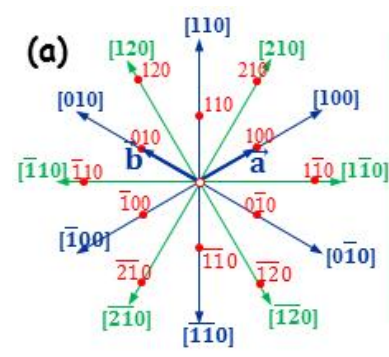

(f)

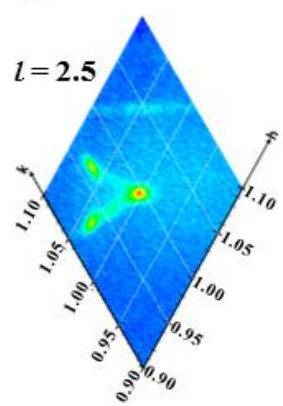

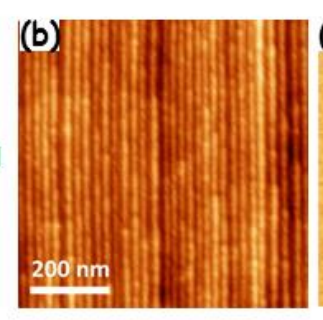

(g)
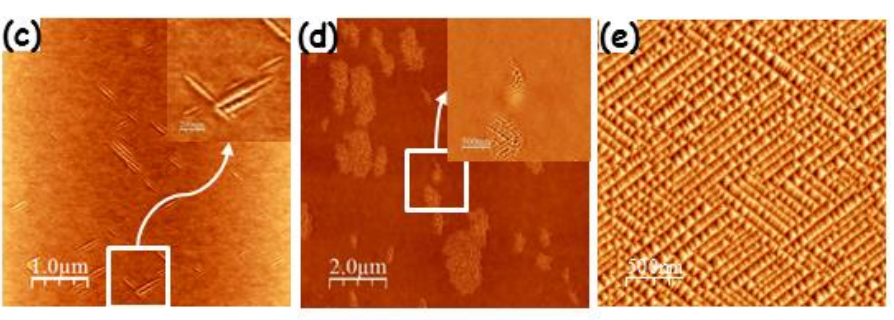
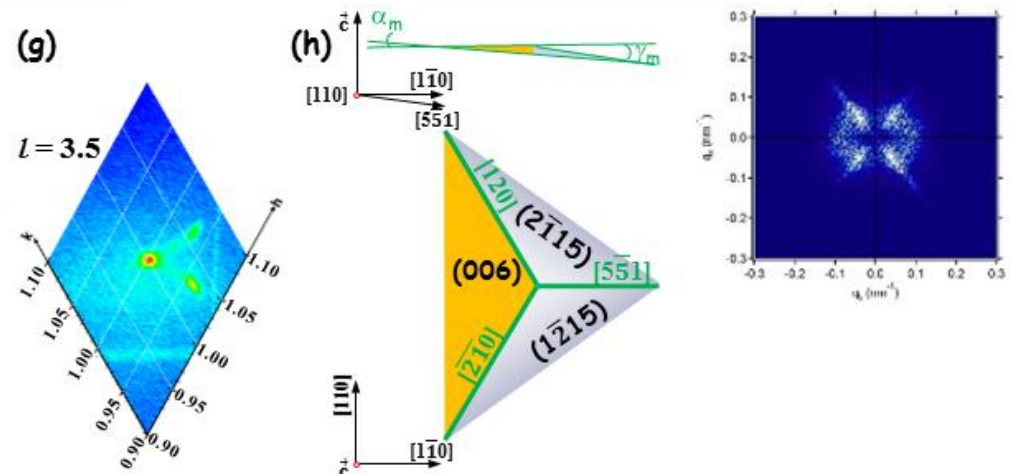

Figure 2 Topography of a nanoscaled Vasarely surface after isothermal treatment at $1250{ }^{\circ} \mathrm{C}$ under oxygen. (a) Sapphire crystallographic directions belonging to the (006) planes. Appearance of the $2 \mathrm{D}$ ordering evaluated by AFM, after $8 \mathrm{~h} \mathrm{(b);} 9 \mathrm{~h} \mathrm{(c);} 10 \mathrm{~h}$ (d) and $32 \mathrm{~h}$ (e) (insert: the corresponding 2D-Fourier transform); (f) and (g) 2Dreciprocal space maps orthogonal to the $\mathrm{c}^{*}$ axis of the $113 \mathrm{RSN}$ at 0.5 reciprocal lattice unit respectively below and above the center of this node for the sample heat treated for $32 \mathrm{~h}$; (h) Geometrical description of the respective arrangement of the corresponding diffracting facets of the same sample.

3a and ref. [19]). A longer thermal treatment induces the modification of the steps that present a zigzag pattern made of an alternation of [210] and [120] segments, respecting the surface symmetry (see fig. 3b). The complete description of the $1 \mathrm{D}$ to $2 \mathrm{D}$ transition process that could be related to meandering $[24,25]$ of the steps is out of the scope of this article. Nevertheless, during this facet reorganization process, the overall surface step density, fixed by the miscut angle $\alpha_{m}$, is kept constant (i.e. new steps cannot be created or destroyed) and the average step direction is conserved to maintain the global macroscopic surface orientation. Moreover, the steps cannot cross each other during the process. Therefore, the steps collectively organize in new zig-zag directions creating new facets along the [110] direction. According to this scenario, 
the surface is now composed by $(006),(2, \overline{1}, 15)$ and $(1, \overline{2}, 15)$ facets. Further thermal treatment induces the organization of the surface towards a 2D periodic system, as shown in fig. $3 \mathrm{c}$. In this case, the facets on the surface organize in a symmetric way with respect to the [110] direction. The surface of the sapphire crystal is therefore composed by a periodic tiling of diamond-shaped (006) facets separated by $(2 \overline{1} 15)$ and $(1 \overline{2} 15)$ parallelogram-shaped facets (see fig 3d). Actually, there is a striking similarity between the schematic representation of the surface drawn in fig. 3c-d and the AFM image reported in fig. $3 \mathrm{e}$.

The centered rectangular cell associated to the $\mathrm{cm}$ plane group [26] is superimposed over the drawing displayed in fig. $3 \mathrm{~d}$ (in green lines and red nodes). Of course, as shown hereafter, depending on the duration of the thermal treatment, the area of the facets evolves and thus the

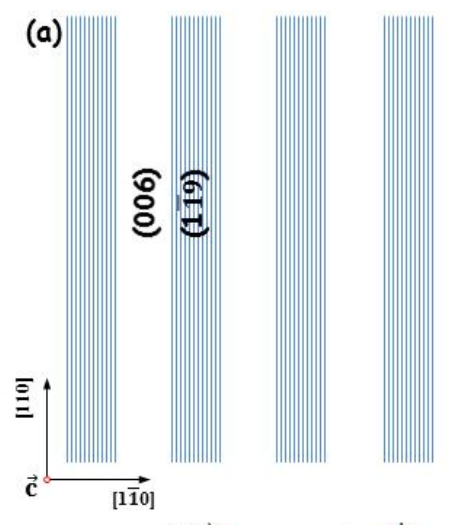

(d)

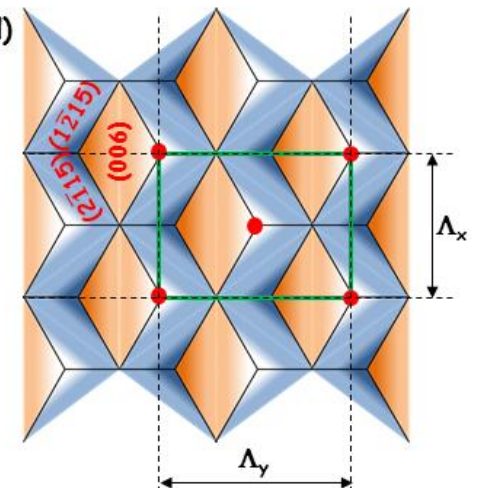

(b)

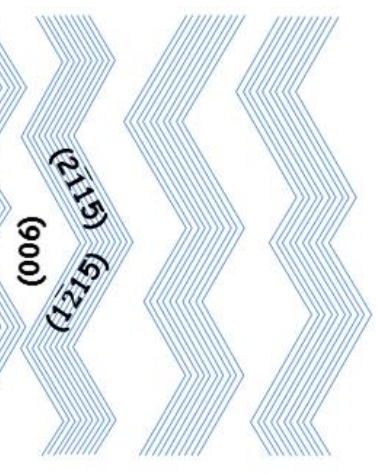

(c)

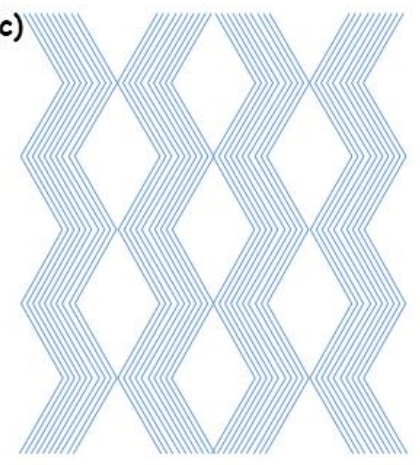

(e)

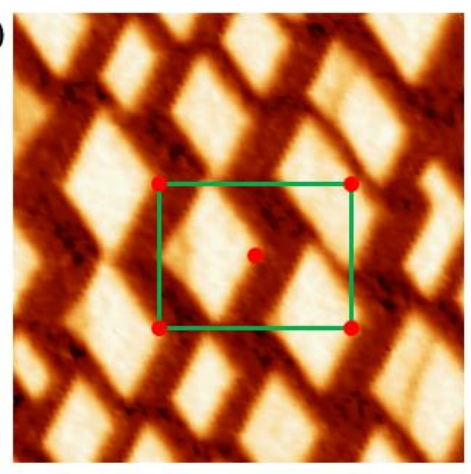

Figure 3 From 1D to 2D self-organization. (a) Modelling of the 1D ordered surface obtained after a thermal treatment of 8 hours; (b) formation of ( $2 \overline{\mathbf{1}} 15)$ and $(1 \overline{\mathbf{2}} 15)$ facets alternated with (006) facets without specific order; (c) closepacked ordering of the $\{2 \overline{\mathbf{1}} 15\}$ facets; (d) modelling of the 2D surface made of diamond-shaped (006) facets separated by $(2 \overline{\mathbf{1}} 15)$ and $(\overline{\mathbf{1}} 215)$ parallelogram-shaped steps; (e) AFM image of the surface recorded after a thermal treatment of 32 hours under oxygen.

dimensions of this cell, which correspond to the $\Lambda_{\mathrm{x}}$ and $\Lambda_{\mathrm{y}}$ periods of the tiling, change accordingly. These values were determined through grazing incidence small-angle X-ray 
scattering (GISAXS) 3D mapping of the reciprocal space [27] by collecting series of $\left(\mathrm{q}_{\mathrm{y}}, \mathrm{q}_{\mathrm{z}}\right)$ GISAXS maps for different azimuthal angles $\varphi$ by increments of $0.5^{\circ}$. In this way, we were able to reconstruct the full 3D GISAXS map of the sapphire vicinal surface heat-treated for 32 hours (see fig. 4a). We observed that the scattered intensity is concentrated in almost vertical scattering rods located in specific azimuthal directions of the reciprocal space. Thanks to the sample orientation procedure described in Sec. $2, \varphi=0^{\circ}$ corresponds to the azimuthal angle for which the projection of the X-ray primary beam onto the sample is along the [110] sapphire direction. Accordingly, it was possible to determine the crystallographic directions corresponding to these specific azimuths and finally to determine the orientation of the centered rectangular reciprocal cell with respect to the $\mathrm{q}_{\mathrm{x}}$ and $\mathrm{q}_{\mathrm{y}}$ components of the scattering vector (see fig. 4b). It is worth to note that two of the scattering streaks observed in this figure are strictly aligned along the diagonals of this rectangle and thus are parallel to the projections of the $\{2 \overline{1} 15\}$ facets normal onto the $\left(\mathrm{q}_{\mathrm{x}}, \mathrm{q}_{\mathrm{y}}\right)$ plane while the third one is aligned with the $\mathrm{q}_{\mathrm{y}}$ edge and is thus parallel to the projection of the (006) facet normal onto the $\left(\mathrm{q}_{\mathrm{x}}, \mathrm{q}_{\mathrm{y}}\right)$ plane. Moreover, it is striking to note that the main features of the 3D-GISAXS map shown in fig. $4 \mathrm{~b}$ are also present in the Fourier transform of the AFM image reported in fig. 2e, which was obtained by observation of the same sample. However, while the AFM image in fig. 2e probes a surface area of a $2.5 \times 2.5 \mu \mathrm{m}^{2}$, the zone irradiated by the X-ray beam during the GISAXS measurements covers an area of roughly one square centimeter! It means that the whole surface of the sapphire crystal is indeed paved by a close packing of (006) and $\{2 \overline{1} 15\}$ facets arranged into a centered rectangular lattice. Lastly, modelling of the GISAXS maps according to the $\mathrm{cm}$ plane group, 
with a value of $\Lambda_{\mathrm{x}}$ equal to $84.5 \mathrm{~nm}$, effectively reproduces the experimental maps (not shown here).
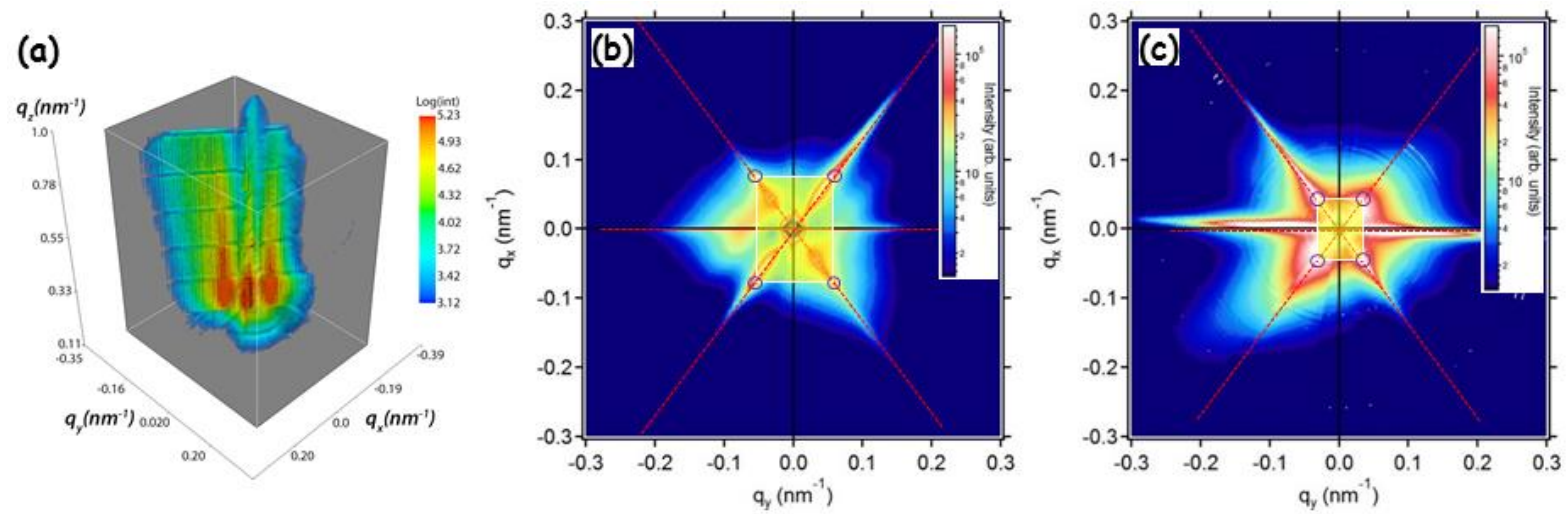

Figure 4 Nanoscaled Vasarely surfaces probed by GISAXS 3D mapping. (a) 3D GISAXS map and (b) projection of this map along the $\mathrm{q}_{\mathrm{z}}$ axis for a vicinal sapphire surface heat-treated for 32 hours at $1250{ }^{\circ} \mathrm{C}$ under oxygen. (c) Projection of the 3D GISAXS intensity along the $\mathrm{q}_{\mathrm{z}}$ axis for a treatment duration of 96 hours. The centered rectangular reciprocal cells are superimposed to the maps reported in the (b) and (c) 2D projections.

According to the schematic representation reported in fig. $3 \mathrm{~d}$, the period $\Lambda_{\mathrm{x}}$ is equal to the longest diagonal of the diamond-shaped (006) facet. Moreover, given the geometrical constraints, the ratio between $\Lambda_{\mathrm{x}}$ and $\Lambda_{\mathrm{y}}$ is

$\frac{\Lambda_{\mathrm{x}}}{\Lambda_{\mathrm{y}}}=\sqrt{3}\left[\frac{\tan \left(\gamma_{m}-\alpha_{m}\right)}{\cos \left(\alpha_{m}\right) \tan \left(\gamma_{m}-\alpha_{m}\right)+\sin \left(\alpha_{m}\right)}\right]=0.752$

In accordance with Eq. 1, the nanoscaled Vasarely surface, which has been obtained through self-organization process after 32 hours of thermal treatment, exhibits two periods equal respectively to $\Lambda_{\mathrm{x}}=84.5 \mathrm{~nm}$ and $\Lambda_{\mathrm{y}}=112 \mathrm{~nm}$. One of the key parameters of these surfaces is of course the control of the $\Lambda_{\mathrm{x}}$ and $\Lambda_{\mathrm{y}}$ values and accordingly the mean surface area of the (006) facets. In fact, it turns out that these values can be tuned by changing the duration of the isothermal treatment. The $\left(\mathrm{q}_{\mathrm{x}}, \mathrm{q}_{\mathrm{y}}\right)$ GISAXS map obtained for a sample heat-treated for 96 hours is reported in fig. $4 \mathrm{c}$. The overall distribution of the scattered intensity is very similar to the one reported in fig. $4 \mathrm{~b}$ corresponding to a treatment duration of 32 hours. This is attested, in particular, by the fact that the centered rectangular reciprocal lattice drawn in fig. $4 \mathrm{~b}$ can also be superimposed to the $\left(\mathrm{q}_{\mathrm{x}}, \mathrm{q}_{\mathrm{y}}\right)$ map obtained after 96 hours of thermal treatment (see fig. $\left.4 \mathrm{c}\right)$. It means that increasing the duration of the thermal treatment causes a homothetic growth of the facets through a coalescence process. From a quantitative point of view, we found that the $\Lambda_{\mathrm{y}}$ 
value increases from $112 \mathrm{~nm}$ after 32 hours of thermal treatment to $188 \mathrm{~nm}$ after 96 hours. The area of the diamond-shaped (006) facets is given by $\frac{\sqrt{3}}{6} \Lambda_{x}^{2}$ and thus according to the equation (1), it increases from about $2075 \mathrm{~nm}^{2}$ after $32 \mathrm{~h}$ of thermal treatment to $5847 \mathrm{~nm}^{2}$ after $96 \mathrm{~h}$, i.e., by a factor of approximately 2.8 close to the duration ratio of 3 . Therefore, this finding indicates that the $2 \mathrm{D}$-coalescence process at $1250{ }^{\circ} \mathrm{C}$ under oxygen results in a quasi-linear isothermal growth in terms of (006) facet mean area.

\section{Conclusions}

To conclude, we have shown that a long duration heat-treatment realized under atmospheric pressure of oxygen of a specific vicinal sapphire surface can induce the formation of nanoscaled Vasarely surfaces made of (006) facets with a tunable size in the range of a few tens of nanometers. Such surfaces might offer considerable possibilities as, for example, substrates for the growth of active devices based on 2D-ordered nanostructures.

\section{Competing interests}

The authors declare no competing financial interests.

\section{Acknowledgments}

This work has been carried out partially within the QMAX Project No. ANR-09-NANO-031 funded by the French National Agency (ANR) in the frame of its program in Nanosciences, Nanotechnologies and Nanosystems (P3N2009). We acknowledge the synchrotron SOLEIL and the ESRF for provision of beamtime at the synchrotron radiation facilities. The authors express their gratitude towards the Limousin Region for financial support of the PhD salaries of $\mathrm{AF}$ and $\mathrm{CM}$.

\section{Author contributions}

E. Thune and R. Guinebretière proposed the scientific subject of self-ordering of sapphire substrates. R. Cavalotti and A. Fakih obtained the first 2D-ordered samples. C. Matringe made the major part of the samples. E. Thune performed all the AFM observations. GISAXS experiments were realized at the BM02 beamline at the ESRF by D. Babonneau, C. Matringe 
and R. Guinebretière with an active contribution of S. Arnaud, N. Blanc and N. Boudet. GIXD measurements were done at the SixS beamline at SOLEIL by C. Matringe, D. Babonneau, R. Guinebretière, A. Coati and Y. Garreau. Quantitative analysis of both GISAXS and GIXD were done by C. Matringe, D. Babonneau, R. Guinebretière, A. Coati and Y. Garreau. R. Guinebretière wrote the manuscript with contributions from all the co-authors.

\section{References}

[1] Barth, J.V.; Costantini, G.; Kern, K. Engineering atomic and molecular nanostructures at surfaces. Nature 2005, 437, 671-679.

[2] Yagi, K.; Minoda, H.; Degawa, M. Step bunching, step wandering and faceting: selforganization at Si surfaces. Surf. Sci. Report 2001, 43, 45-126.

[3] Misbah, C.; Pierre-Louis, O.; Saito, Y. Crystal surfaces in and out of equilibrium: A modern view. Rev. Modern Phys. 2010, 82, 981-1040.

[4] Kim, Y.; Jo, M.H.; Kim, T.C.; Yang, C.W.; Kim, J.W.; Hwang, J.S.; Noh, D.Y.; Kim, N.D.; Chung, J.W. Coarsening kinetics of a spinodally decomposed vicinal Si(111) surface. Phys. Rev. Lett. 2009, 102, 156103.

[5] Rousset, S.; Repain, V.; Baudot, G.; Garreau, Y.; Lecoeur, J. Self-ordering of Au(111) vicinal surfaces and application to nanostructure organized growth. J. Phys. Cond. Matter 2003, 15, S3363-S3392.

[6] Men, F.K.; Liu, F.; Wang, P.J.; Chen, C.H.; Cheng, D.L.; Lin, J.L.; Himpsel, F.J. Selforganized nanoscale pattern formation on vicinal $\mathrm{Si}(111)$ surfaces via two-stage faceting transition. Phys. Rev. Lett. 2002, 88, 096105.

[7] Cuccureddu, F.; Murphy, S.; Shvets, I.V.; Porcu, M.; Zandbergen, H.W.; Sidorov, N.S.; Bolzhko, S.I. Surface morphology of c-plane sapphire ( $\alpha$-alumina) produced by hightemperature anneal. Surf. Sci. 2010, 604, 1294-1299.

[8] Sanchez, F.; Herranz, G.; Infante, I.C.; Fontcuberta, J.; Garcia-Cuenca, M.V.; Ferrater, C.; Varela, M. Critical effects of substrate terraces and steps morphology on the growth mode of epitaxial $\mathrm{SrRuO}_{3}$ films. Appl. Phys. Lett. 2004, 85, 1981-1983.

[9] Nakamura, S. The roles of structural imperfections in InGaN-based blue light-emitting diodes and laser diodes. Science 1998, 281, 956-961.

[10] Koester, R.; Hwang, J.S.; Salomon, D.; Chen, X.; Bougerol, C.; Barnes, J.P.; Dang, D.L.S.; Rigutti, L.; Bugallo, A.L.; Jacopin, G.; Tchernycheva, M.; Durand, C.; Eymery, J. 
M-plane core shell InGaN/GaN multiple-quantum-wells on $\mathrm{GaN}$ wires for electroluminescent devices. Nano Lett. 2011, 11, 43839-43845.

[11] Huang, M.H.; Mao, S.; Feick, H.; Yan, H.Q.; Wu, Y.Y.; Kind, H.; Weber, E.; Russo, R.; Yang, P.D. Room-temperature ultraviolet nanowire nanolasers. Science 2001, 292, 1897-1899.

[12] Eaton, S.W.; Fu, A.; Wong, A.B.; Ning, C.Z.; Yang, P.D. Semiconductor nanowire lasers. Nat. Rev. Mater. 2016, 1, 16028.

[13] Lee G.H. Role of substrate step bunches on the growth behavior of $\mathrm{LiNbO}_{3}$ thin film on $\alpha-\mathrm{Al}_{2} \mathrm{O}_{3}$ substrate. Mater. Sci. Eng. B 2007, 138, 41-45.

[14] Ago, H.; Imamoto, K.; Ishigami, N.; Ohdo, R.; Ikeda, K.I.; Tsuji M. Competition and cooperation between lattice-oriented growth and step-templated growth of aligned carbon nanotubes on sapphire. Appl. Phys. Lett. 2007, 90, 133112.

[15] Bachelet, R.; Cottrino, S.; Nahélou, G.; Coudert, V.; Boulle, A.; Soulestin, B.; Rossignol, F.; Guinebretière R.; Dauger, A. Self-patterned oxide nanostructures grown by post-deposition thermal annealing on stepped surfaces. Nanotechnology 2007, 18, 015301.

[16] Boulle, A.; Kilburger, S.; Di Bin, P.; Millon, E.; Di Bin, C., Guinebretière, R., Bessaudou A. Structural and optical characterizations of high quality $\mathrm{LiNbO}_{3}$ epitaxial films grown by pulsed laser deposition. J. Phys. D: Appl. Phys. 2009, 42, 145403.

[17] Aoki, R.; Arakawa, T.; Misawa, N.; Tero, R.; Urisu, T.; Takeuchi, A.; Ogino, T. Immobilization of protein molecules on step-controlled sapphire surfaces. Surf. Sci. 2007, $601,4915-4921$.

[18] Thune, E.; Fakih, A.; Matringe, C.; Babonneau, D.; Guinebretière, R. Understanding of one dimensional ordering mechanisms at the (001) sapphire vicinal surface. J. Appl. Phys. 2017, 121, 015301.

[19] Matringe, C.; Fakih, A.; Thune, E.; Babonneau, D.; Arnaud, S.; Blanc, N.; Boudet, N.; Guinebretière, R. Symmetric faceting of a sapphire vicinal surface revealed by Grazing Incidence Small-Angle X-ray Scattering 3D mapping. Appl. Phys. Lett. 2017, 111, 031601.

[20] Horcas, I.; Fernandez, R.; Gomez-Rodriguez, J. M.; Colchero, J.; Gomez-Herrero, J., and Baro, A. M. WSXM: A software for scanning probe microscopy and a tool for nanotechnology. Rev. Sci. Instrum. 2007, 78, 013705.

[21] Chahine, G.A.; Blanc, N.; Arnaud, S.; Geuser, F. de; Guinebretière, R.; Boudet, N. Advanced non-destructive in situ characterization of metals at the French CRG D2AM / BM02 beamline at the ESRF. Metals 2019, 9, 352. 
[22] Babonneau, D. FitGISAXS: software package for modelling and analysis of GISAXS data using IGOR Pro. J. Appl. Cryst. 2010, 43, 929-936.

[23] Robinson, I.K. Crystal truncation rods and surface roughness. Phys. Rev. B 1986, 33, $3830-3836$

[24] Pimpinelli, A.; Villain, J.; Wolf, D.E.; Metois, J.J.; Heyraud, J.C.; Elkinani, I.; Uimin, G. Equilibrium step dynamics on vicinal surfaces. Surf. Sci. 1993, 295, 143-153.

[25] Jeong, H.C.; Williams, E.D. Steps on surfaces: experiment and theory. Surf. Sci. Rep. 1999, 34, 171-294.

[26] International Tables for Crystallography Volume A: Space-group symmetry, Edited by Th. Hahn 2011.

[27] Babonneau, D.; Vandenhecke, E.; Camelio, S. Formation of nanoripples on amorphous alumina thin films during low-energy ion-beam sputtering: Experiments and simulations Phys. Rev. B 2017, 95, 085412. 